\title{
Effect of Dietary Lead Exposure on Hematological Parameters and Their Alleviation by Antioxidants in Broilers
}

\author{
Ritesh Jaiswal ${ }^{1}$, S. L. Ali ${ }^{1}$, S. Roy ${ }^{1}$, O. P. Dinani ${ }^{1}$ and Sudhir Kumar Jaiswal ${ }^{2 *}$ \\ ${ }^{1}$ College of Veterinary Science and A. H. Anjora, Durg, Chhattisgarh (491 001), India \\ ${ }^{2}$ Division of Poultry Science, Indian Veterinary Research Institute, Izatnagar, Bareilly, Uttar Pradesh (243 122), India
}

\section{Corresponding Author}

Piyush Pradhan

e-mail: sjsudhirjaiswal009@gmail.com

\author{
Article History \\ Manuscript No. AR1778 \\ Received in $15^{\text {th }}$ Jan, 2017 \\ Received in revised form $6^{\text {th }}$ Feb, 2017 \\ Accepted in final form $7^{\text {th }}$ Feb, 2017
}

\begin{abstract}
A total number of 126 day old broiler chicks were randomly assigned into 6 treatments groups A, B, C, D, E and F. The birds of group A were kept as control, while group B received lead acetate @ $200 \mathrm{mg} \mathrm{kg}^{-1}$ alone, whereas, birds of group C, D, E and F received Lead acetate @ $200 \mathrm{mg} \mathrm{kg}^{-1}+$ Ascorbic acid @ $200 \mathrm{mg} \mathrm{kg}^{-1}$, lead acetate @ $200 \mathrm{mg} \mathrm{kg}^{-1}$ along with $\alpha$-tocopherol @ $100 \mathrm{mg} \mathrm{kg}^{-1}+$ Selenium @ $0.1 \mathrm{mg} \mathrm{kg}^{-1}$, Lead acetate @ $200 \mathrm{mg} \mathrm{kg}^{-1}+\mathrm{DL}-$ methionine @ $100 \mathrm{mg} \mathrm{kg}^{-1}$ and Lead acetate @ $200 \mathrm{mg} \mathrm{kg}^{-1}+$ methanolic extract of Cissus quadrangularis (CQE) @ $400 \mathrm{mg} \mathrm{kg}^{-1}$ respectively. In group-B TEC, hemoglobin and MCV declined significantly $(p<0.05)$ as compared to control on day 21. At day 42 significant $(p<0.05)$ decrease in $\mathrm{Hb}, \mathrm{PCV}$ and MCV was recorded in lead treated group. Treatment with Vit-E and Se (group-D) was found effective in restoration of TEC and $\mathrm{Hb}$. Evaluation of leucocyte parameters revealed no significant $(p<0.05)$ difference in total and differential leucocytic count on day 21 and 42 . However, significant $(p<0.05)$ increase in lymphocyte count was recorded in lead exposed group as compared to other groups. Vit-E @ $100 \mathrm{mg} \mathrm{kg}^{-1}$ and Se @ $0.1 \mathrm{mg} \mathrm{kg}^{-1}$ having highest efficacy against lead intoxication followed by Vitamin-C@200 mg kg-1, DL-methionine @ $100 \mathrm{mg} \mathrm{kg}^{-1}$ and methanolic extract of CQE @ $400 \mathrm{mg} \mathrm{kg}^{-1}$ to alleviate lead toxicosis upto the level of $200 \mathrm{mg} \mathrm{kg}^{-1}$ in broiler diet.
\end{abstract}

Keywords: Lead, hematological parameters, antioxidants, broilers, Cissus quadrangularis

\section{Introduction}

Lead a potent heavy environmental pollutant is naturally occurring elements that has been mobilized and redistributed to a large extent in the environment by industrialization and urbanization process. Berg et al. (1980) stated that lead contamination of environment due its prolonged exposure and slow rate of elimination (Demichele, 1984; Ercal et al., 2001). Lead is a common source of poisoning in domestic animals all over the world (Khan et al., 2008). A good source of lead contamination of poultry feed is bone and blood meals, majority of which comes from cattle. However, in cattle, highest lead accumulation has been reported to occur in bones (Heaney, 2000). A main effect of lead poisoning is anemia, which consequences from inhibition of the heme synthesizing enzymes with concurrent elevation of protoporphyrin (Lee, 1981). This statement was further buttressed by Osweiler (1996) who reported that lead slows down haemosynthesis through inhibition of enzymes. Ascorbic acid (AA) is a wellknown antioxidant vitamin involved in several biochemical processes in biological systems. This vitamin breaks the chain of lipid peroxidation in cell membranes and scavenges free radicals such as reactive oxygen species (Carr \& Frei, 1999; Kucuk et al., 2003). Lead has reached to such a harmful level that it can affects the growth, productivity and health of poultry as well as animals due to cumulative poisoning. But poultry has not been studied to the desired extent in comparison with other farm animals. With a view to study the effect of lead on hematological parameters of broilers, present investigation was under taken. We also aimed to determine whether the effects of lead, attributed on hematological parameters, could be reversed by adding antioxidants.

\section{Materials and Methods}

The present study was undertaken in the Department of Veterinary Medicine, College of Veterinary Science and A.H., Anjora, Durg (Chhattisgarh), India in the year of 2010. In this study 126 day old broiler chicks (Ven-Cobb strain, IB Group, Rajnandgaon, Chhattisgarh) were randomly divided into six treatment groups ( $n=21)$ as A, B, C, D, E and F and all treatments were given to chicks on the basis of $\mathrm{kg}^{-1}$ basal diet daily for the period of 42 days. The birds of group-A were kept as healthy 
control received only basal diet, birds of group- $B$ received lead acetate alone @ $200 \mathrm{mg} \mathrm{kg}^{-1}$ where as birds of group-C received lead acetate @ $200 \mathrm{mg} \mathrm{kg}^{-1}$ along with ascorbic acid @ $200 \mathrm{mg} \mathrm{kg}^{-1}$, group-D got vit-E @ 100 mg kg-1 and Se @ 0.1 $\mathrm{mg} \mathrm{kg}^{-1}$, group-E received DL-methionine @ $100 \mathrm{mg} \mathrm{kg}^{-1}$ and methanolic extract Cissus quadrangularis (CQE) @ $400 \mathrm{mg} \mathrm{kg}^{-1}$ was given to group-F respectively for 42 days. About 5-6 ml of blood sample were collected aseptically from metatarsal vein of birds by using 22 gauge needle in the sterilized, neat and clean plastic vials containing heparin anticoagulant @ 0.1 $\mathrm{ml} 10 \mathrm{ml}^{-1}$ of blood (Heparin as a 1\% solution 1,000 USP units $\mathrm{ml}^{-1}$ ) on $21^{\text {st }}$ and $42^{\text {nd }}$ day of experiment in the morning hours from 6 broilers that were randomly chosen from each group. Haematology was performed to evaluate the effect of lead on blood parameters. The following blood parameters were evaluated at 21 and 42 days by using Automated Haematology Blood Cell Counter of Melet Schloesing Laboratories of Sussi France (MS9). Total Erythrocyte count (TEC in Million $\mu^{-1}$ ), Haemoglobin ( $\mathrm{Hb}$ in $\mathrm{g} \%$ or $\mathrm{g} \mathrm{dl}^{-1}$ ), Packed cell volume (PCV in \%), Mean corpuscular value (MCV in fl), Mean corpuscular haemoglobin ( $\mathrm{MCH}$ in pg), Mean corpuscular haemoglobin concentration ( $\mathrm{MCHC}$ in $\mathrm{g} \mathrm{dl}^{-1}$ ), Total Leukocyte count (TLC in Thousand $\mu^{-1}$ ), Differential Leukocyte count (DLC in \%).The data were analyzed statistically using analysis of variance with one way classification followed by Dancan's Multiple Range Test (DMRT) to find out the significance of difference between mean values of different groups as per the procedure outlined by Snedecor and Cochran (1994).

\section{Results and Discussion}

\subsection{Haematological changes}

The results of haematological profile are summarized in the Table 1 for day 21 and Table 2 for day 42 and comparison between day 21 and 42 are presented in Table 3.

\subsubsection{Total erythrocytic count (TEC)}

The obtained values in group $A$ (control), B, C, D, E and F were $1.28 \pm 0.04,0.93 \pm 0.01,1.42 \pm 0.04,1.16 \pm 0.07,1.14 \pm 0.06$ and

\begin{tabular}{|c|c|c|c|c|c|c|}
\hline \multirow{2}{*}{$\begin{array}{l}\text { Haematological } \\
\text { parameters }\end{array}$} & \multicolumn{6}{|c|}{ Various treatment groups showing mean $\pm S E$ values } \\
\hline & $A$ & B & $\mathrm{C}$ & D & $\mathrm{E}$ & $\mathrm{F}$ \\
\hline $\operatorname{TEC}\left(\left.10^{6} \mu\right|^{-1}\right)$ & $1.28 \pm 0.04^{\mathrm{bc}}$ & $0.93 \pm 0.01^{\mathrm{a}}$ & $1.42 \pm 0.04^{c}$ & $1.16 \pm 0.07^{b}$ & $1.14 \pm 0.06^{b}$ & $1.23 \pm 0.67^{b}$ \\
\hline $\mathrm{Hb}\left(\mathrm{gdl}^{-1}\right)$ & $9.77 \pm 0.36^{b}$ & $7.43 \pm 0.13^{\mathrm{a}}$ & $10.44 \pm 0.23^{b c}$ & $11.70 \pm 1.29^{c}$ & $9.60 \pm 0.09^{b}$ & $9.33 \pm 0.30^{b}$ \\
\hline PCV (\%) & $14.47 \pm 0.41^{\mathrm{bc}}$ & $9.83 \pm 0.15^{c}$ & $15.43 \pm 0.29^{c}$ & $13.27 \pm 0.79^{b}$ & $13.22 \pm 0.62^{\mathrm{b}}$ & $13.90 \pm 0.71^{\mathrm{bc}}$ \\
\hline $\operatorname{MCV}(f l)$ & $113.09 \pm 0.61^{b c}$ & $105.35 \pm 1.02^{\mathrm{a}}$ & $109.25 \pm 2.11^{\mathrm{ab}}$ & $114.28 \pm 1.64^{\mathrm{bc}}$ & $116.33 \pm 3.44^{c}$ & $113.13 \pm 1.25^{\mathrm{bc}}$ \\
\hline $\mathrm{MCH}(\mathrm{pg})$ & $76.41 \pm 2.11^{\mathrm{a}}$ & $79.53 \pm 0.79^{\mathrm{a}}$ & $74.91 \pm 1.80^{\mathrm{a}}$ & $101.64 \pm 11.11^{\mathrm{b}}$ & $85.26 \pm 4.34^{\mathrm{a}}$ & $76.51 \pm 2.30^{\mathrm{a}}$ \\
\hline $\mathrm{MCHC}\left(\mathrm{g} \mathrm{dl}^{-1}\right)$ & $67.54 \pm 1.60^{\mathrm{a}}$ & $75.53 \pm 1.04^{\mathrm{a}}$ & $68.74 \pm 2.42^{\mathrm{a}}$ & $89.46 \pm 10.73^{b}$ & $73.26 \pm 3.10^{\mathrm{a}}$ & $67.66 \pm 2.10^{\mathrm{a}}$ \\
\hline $\operatorname{TLC}\left(10^{3} \mathrm{dl}^{-1}\right)$ & $139.88 \pm 5.49^{b c}$ & $146.91 \pm 4.00^{c}$ & $127.91 \pm 1.98^{\mathrm{ab}}$ & $122.22 \pm 10.66^{a}$ & $138.41 \pm 0.61^{\mathrm{bc}}$ & $140.04 \pm 2.50^{\mathrm{bc}}$ \\
\hline Lymphocyte(\%) & $33.10 \pm 1.84^{\mathrm{a}}$ & $52.47 \pm 1.28^{c}$ & $31.27 \pm 0.17^{a}$ & $41.87 \pm 3.62^{\mathrm{b}}$ & $35.77 \pm 0.80^{\mathrm{a}}$ & $35.27 \pm 02.28^{a}$ \\
\hline Monocyte (\%) & $6.60 \pm 0.27^{\mathrm{ab}}$ & $7.06 \pm 0.11^{\mathrm{bc}}$ & $6.37 \pm 0.09^{\mathrm{a}}$ & $7.30 \pm 0.15^{c}$ & $7.00 \pm 0.10^{\mathrm{bc}}$ & $6.67 \pm 0.21^{\mathrm{ab}}$ \\
\hline Heterophil (\%) & $59.16 \pm 2.24^{b c}$ & $54.63 \pm 0.97^{\mathrm{ab}}$ & $60.90 \pm 0.43^{c}$ & $49.70 \pm 3.22^{\mathrm{a}}$ & $55.23 \pm 0.65^{\mathrm{abc}}$ & $56.56 \pm 2.32^{\mathrm{bc}}$ \\
\hline Eosinophil (\%) & $1.00 \pm 0.36^{\mathrm{a}}$ & $1.67 \pm 0.33^{a}$ & $1.17 \pm 0.31^{\mathrm{a}}$ & $1.00 \pm 0.51^{\mathrm{a}}$ & $1.33 \pm 0.42^{\mathrm{a}}$ & $1.17 \pm 0.31^{\mathrm{a}}$ \\
\hline Basophil (\%) & $0.50 \pm 0.22^{\mathrm{a}}$ & $0.67 \pm 0.21^{\mathrm{a}}$ & $0.33 \pm 0.21^{\mathrm{a}}$ & $0.50 \pm 0.22^{\mathrm{a}}$ & $0.67 \pm 0.21^{\mathrm{a}}$ & $0.56 \pm 0.21^{\mathrm{a}}$ \\
\hline
\end{tabular}

a-c Mean values bearing different superscripts within a row differ significantly $(p<0.05)$

$1.23 \pm 0.6710^{6} \mu \mathrm{l}^{-1}$ respectively on 21 days, while on day 42 TEC values in group A (control), B, C, D, E and F was 2.30 \pm 0.11 , $1.82 \pm 0.11,2.06 \pm 0.30,2.45 \pm 0.13,2.44 \pm 0.26$ and $2.44 \pm 0.27$ $10^{6} \mathrm{Hl}^{-1}$ respectively. A significant higher $(p<0.05)$ value of RBC count was recorded in control and other treatment group as compared to lead treated groups on day 21. However comparison among group $C, D, E$ and $F$ revealed, significant $(p<0.05)$ difference in the RBC count of group $C$ from the others treatment groups but group $D, E$ and $F$ was not significantly $(p>0.05)$ comparable to each other. Though on day 42 TEC values were significantly $(p<0.05)$ increased on day 21 onwards in all groups but at the end of the study lead treated group showed no significant $(p>0.05)$ changes from the control (group A) and other treatment groups. RBC count of different treatment group was comparable to each other on day 42 .

\subsubsection{Haemoglobin $(\mathrm{Hb})$}

Mean value of hemoglobin was significantly higher $(p<0.05)$ in control $\left(9.77 \pm 0.36 \mathrm{~g} \mathrm{dl}^{-1}\right)$ and other treatment group $C$ $\left(10.44 \pm 0.23 \mathrm{~g} \mathrm{dl}^{-1}\right), \mathrm{D}\left(11.70 \pm 1.29 \mathrm{~g} \mathrm{dl}^{-1}\right), \mathrm{E}\left(9.60 \pm 0.09 \mathrm{~g} \mathrm{dl}^{-1}\right)$ and $F\left(9.33 \pm 0.30 \mathrm{~g} \mathrm{dl}^{-1}\right)$ as compared to lead treated group $B$ $\left(7.43 \pm 0.13 \mathrm{~g} \mathrm{dl}^{-1}\right)$ on day 21 . Comparison among the different treatment groups showed highest $\mathrm{Hb}$ level in group $\mathrm{D}$ followed by group C, A, E and F. On day 42 lead treated group ( $8.90 \pm 0.22$ $\left.\mathrm{g} \mathrm{dl}^{-1}\right)$ showed significant $(P<0.05)$ reduction in $\mathrm{Hb}$ as compared to group A $\left(13.30 \pm 0.64 \mathrm{~g} \mathrm{dl}^{-1}\right), \mathrm{D}\left(14.66 \pm 0.90 \mathrm{~g} \mathrm{dl}^{-1}\right)$ and $E$ $\left(15.60 \pm 2.09 \mathrm{~g} \mathrm{dl}^{-1}\right)$ and non-significant $(p>0.05)$ reduction from group $C\left(12.67 \pm 1.71 \mathrm{~g} \mathrm{dl}^{-1}\right)$ and $\mathrm{F}\left(12.71 \pm 0.98 \mathrm{~g} \mathrm{dl}^{-1}\right)$. 
International Journal of Bio-resource and Stress Management 2017, 8(1):110-115

\begin{tabular}{|c|c|c|c|c|c|c|}
\hline \multirow{2}{*}{$\begin{array}{l}\text { Haematological } \\
\text { parameters }\end{array}$} & \multicolumn{6}{|c|}{ Various treatment groups showing mean $\pm S E$ values } \\
\hline & A & B & C & $\mathrm{D}$ & $\mathrm{E}$ & $\mathrm{F}$ \\
\hline $\operatorname{TEC}\left(10^{6} \mu \mathrm{l}^{-1}\right)$ & $2.30 \pm 0.11^{\mathrm{a}}$ & $1.82 \pm 0.11^{\mathrm{a}}$ & $2.06 \pm 0.30^{\mathrm{a}}$ & $2.45 \pm 0.13^{a}$ & $2.44 \pm 0.26^{\mathrm{a}}$ & $2.44 \pm 0.27^{a}$ \\
\hline $\mathrm{Hb}\left(\mathrm{g} \mathrm{dl}^{-1}\right)$ & $13.30 \pm 0.64^{b}$ & $8.90 \pm 0.22^{\mathrm{a}}$ & $12.67 \pm 1.71^{\mathrm{ab}}$ & $14.66 \pm 0.90^{b}$ & $15.60 \pm 2.09^{b}$ & $12.71 \pm 0.98^{\mathrm{ab}}$ \\
\hline PCV (\%) & $24.86 \pm 1.38^{b}$ & $16.13 \pm 0.52^{\mathrm{a}}$ & $22.28 \pm 3.09^{\mathrm{ab}}$ & $26.42 \pm 1.39^{b}$ & $26.30 \pm 2.99^{b}$ & $26.09 \pm 2.81^{\mathrm{b}}$ \\
\hline $\operatorname{MCV}(\mathrm{fl})$ & $107.87 \pm 1.60^{b}$ & $89.91 \pm 3.73^{a}$ & $108.87 \pm 2.30^{b}$ & $107.56 \pm 0.86^{b}$ & $107.35 \pm 1.42^{b}$ & $107.13 \pm 0.47^{b}$ \\
\hline $\mathrm{MCH}(\mathrm{pg})$ & $57.83 \pm 0.61^{\mathrm{ab}}$ & $49.75 \pm 2.49^{\mathrm{a}}$ & $62.06 \pm 2.41^{b}$ & $59.58 \pm 0.78^{\mathrm{ab}}$ & $63.03 \pm 1.79^{b}$ & $55.36 \pm 7.00^{\mathrm{ab}}$ \\
\hline $\operatorname{MCHC}\left(\mathrm{g} \mathrm{dl}^{-1}\right)$ & $53.65 \pm 0.42^{\mathrm{a}}$ & $55.29 \pm 1.20^{\mathrm{a}}$ & $56.96 \pm 1.56^{a}$ & $55.41 \pm 0.82^{\mathrm{a}}$ & $58.69 \pm 1.37^{a}$ & $51.63 \pm 6.41^{\mathrm{a}}$ \\
\hline $\operatorname{TLC}\left(10^{3} \mathrm{dl}^{-1}\right)$ & $98.27 \pm 1.37^{\mathrm{ab}}$ & $83.97 \pm 2.36^{a}$ & $106.59 \pm 9.94^{\mathrm{ab}}$ & $119.46 \pm 0.80^{b}$ & $102.25 \pm 10.54^{\mathrm{ab}}$ & $94.56 \pm 10.70^{a}$ \\
\hline Lymphocyte(\%) & $22.83 \pm 1.61^{\mathrm{a}}$ & $20.65 \pm 0.66^{a}$ & $27.63 \pm 3.58^{\mathrm{a}}$ & $28.63 \pm 0.48^{\mathrm{a}}$ & $23.00 \pm 2.76^{\mathrm{a}}$ & $22.46 \pm 3.10^{\mathrm{a}}$ \\
\hline Monocyte (\%) & $5.13 \pm 0.32^{\mathrm{a}}$ & $6.13 \pm 0.21^{\mathrm{a}}$ & $5.70 \pm 0.64^{\mathrm{a}}$ & $5.16 \pm 0.31^{\mathrm{a}}$ & $5.17 \pm 0.53^{\mathrm{a}}$ & $4.75 \pm 0.70^{\mathrm{a}}$ \\
\hline Heterophil (\%) & $70.54 \pm 1.42^{\mathrm{a}}$ & $71.86 \pm 1.06^{\mathrm{a}}$ & $65.32 \pm 3.78^{\mathrm{a}}$ & $64.78 \pm 0.64^{a}$ & $70.00 \pm 2.71^{\mathrm{a}}$ & $71.12 \pm 4.14^{\mathrm{a}}$ \\
\hline Eosinophil (\%) & $1.00 \pm 0.36^{a}$ & $1.67 \pm 0.33^{\mathrm{a}}$ & $0.83 \pm 0.31^{\mathrm{a}}$ & $0.67 \pm 0.21^{\mathrm{a}}$ & $1.17 \pm 0.31^{\mathrm{a}}$ & $1.17 \pm 0.40^{\mathrm{a}}$ \\
\hline Basophil (\%) & $0.50 \pm 0.22^{\mathrm{a}}$ & $0.67 \pm 0.21^{\mathrm{a}}$ & $0.50 \pm 0.22^{\mathrm{a}}$ & $0.33 \pm 0.21^{\mathrm{a}}$ & $0.67 \pm 0.33^{\mathrm{a}}$ & $0.50 \pm 0.22^{\mathrm{a}}$ \\
\hline
\end{tabular}

a-c Mean values bearing different superscripts within a row differ significantly $(p<0.05)$

\subsubsection{Packed cell volume (PCV)}

Mean value of packed cell volume (PCV) was no significantly $(p>0.05)$ higher in group A $(14.47 \pm 0.41 \%)$, group C $(15.43 \pm 0.29 \%)$ and group $F(13.90 \pm 0.71 \%)$, but significantly $(p<0.05)$ higher in group $D(13.27 \pm 0.79 \%)$ and group E $(13.22 \pm 0.62 \%)$ as compared to lead treated group B $(9.83 \pm 0.15 \%)$ on day 21 . Comparison among the different treatment groups showed highest PCV level in group C followed by group A, F, D and E. Although on 42 days PCV values were significantly $(p<0.05)$ increased from the day 21 in all groups. But on day 42 lead treated group $(16.13 \pm 0.52 \%)$ showed significant $(p<0.05)$ reduction of PCV from the group A $(24.86 \pm 1.38 \%), D(26.42 \pm 1.39 \%), E(26.30 \pm 2.99 \%)$ and $F$ $(26.09 \pm 2.81 \%)$ but no-significant $(p>0.05)$ reduction from group C (22.28 $\pm 3.09 \%)$.

\subsubsection{Mean corpuscular volume (MCV)}

The mean values of MCV are significantly $(p<0.05)$ higher values were obtained in control group A $(113.09 \pm 0.61 \mathrm{fl}), \mathrm{D}$ (114.28 $\pm 1.64 \mathrm{fl}), \mathrm{E}(116.33 \pm 3.44 \mathrm{fl})$ and $F(113.13 \pm 1.25 \mathrm{fl})$ but non-significantly $(p>0.05)$ higher in group $C(109.25 \pm 2.11 \mathrm{fl})$ as compared to lead treated group B $(105.35 \pm 1.02 \mathrm{fl})$ on day 21. Comparison among the different treatment groups on day 21 showed highest MCV level in group-E followed by group $\mathrm{D}>\mathrm{F}>\mathrm{A}>\mathrm{C}$. Although on day $42 \mathrm{MCV}$ values were significantly $(p<0.05)$ increased from the day 21 in all groups except lead treated group which showed significantly $(p>0.05)$ lower MCV values from day 21 till end of the study. On day 42 lead treated group $(89.91 \pm 3.73 \mathrm{fl})$ showed significant $(p>0.05)$ reduction of PCV from the group A $(107.87 \pm 1.60 \mathrm{fl}), \mathrm{C}(108.87 \pm 2.30 \mathrm{fl})$, $D(107.56 \pm 0.86 \mathrm{fl}), E(107.35 \pm 1.42 \mathrm{fl})$ and $F(107.13 \pm 0.47 \mathrm{fl})$.

\subsubsection{Mean corpuscular haemoglobin $(\mathrm{MCH})$}

The mean $\mathrm{MCH}$ values were non-significant $(p>0.05)$ comparable in group A $(76.41 \pm 2.11 \mathrm{pg}), \mathrm{B}(79.53 \pm 0.79 \mathrm{pg}), \mathrm{C}$
(74.91 $\pm 1.80 \mathrm{pg}), \mathrm{E}(85.26 \pm 4.34 \mathrm{pg})$ and group $\mathrm{F}(76.51 \pm 2.30$ pg) but significantly $(p<0.05)$ higher in group $D(101.64 \pm 11.11$ $\mathrm{pg}$ ) as compared to lead treated group on day 21. Comparison among the different treatment groups on day 21 showed highest $\mathrm{MCH}$ level in group-D while others showed nonsignificant $(p>0.05)$ changes. Although on 42 days $\mathrm{MCH}$ values were significantly $(p<0.05)$ decreased from the day 21 in all groups. But on day 42 lead treated group B $(49.75 \pm 2.49 \mathrm{pg})$ showed non-significant $(p>0.05)$ reduction of $\mathrm{MCH}$ from the group A $(57.83 \pm 0.61 \mathrm{pg}), \mathrm{D}(59.58 \pm 0.78 \mathrm{pg}), \mathrm{F}(55.36 \pm 7.00$ pg), but significant $(p<0.05)$ reduction of $\mathrm{MCH}$ from group $\mathrm{C}$ $(62.06 \pm 2.41 \mathrm{pg})$ and $\mathrm{E}(63.03 \pm 1.79 \mathrm{pg})$.

\subsubsection{Mean corpuscular haemoglobin concentration (MCHC)}

The mean MCHC values were non-significantly $(p>0.05)$ decreased in lead treated group $B\left(75.53 \pm 1.04 \mathrm{~g} \mathrm{dl}^{-1}\right)$ from control group A $\left(67.54 \pm 1.60 \mathrm{~g} \mathrm{dl}^{-1}\right), \mathrm{C}\left(68.74 \pm 2.42 \mathrm{~g} \mathrm{dl}^{-1}\right), \mathrm{E}$ $\left(73.26 \pm 3.10 \mathrm{~g} \mathrm{dl}^{-1}\right)$ and $\mathrm{F}\left(67.66 \pm 2.10 \mathrm{~g} \mathrm{dl}^{-1}\right)$ but significant $(p<0.05)$ reduction was observed in group $\mathrm{D}(89.46 \pm 10.73 \mathrm{~g}$ $\left.\mathrm{dl}^{-1}\right)$ on day 21. Comparative changes in different groups from day 21 to 42 showed significant $(p<0.05)$ alteration. On day 42 lead treated group $B\left(55.29 \pm 1.20 \mathrm{~g} \mathrm{dl}^{-1}\right)$ showed non-significant $(p>0.05)$ reduction of $\mathrm{MCHC}$ from the group $\mathrm{C}(56.96 \pm 1.56 \mathrm{~g}$ $\left.\mathrm{dl}^{-1}\right), \mathrm{D}\left(55.41 \pm 0.82 \mathrm{~g} \mathrm{dl}^{-1}\right), \mathrm{E}\left(58.69 \pm 1.37 \mathrm{~g} \mathrm{dl}^{-1}\right)$ and group $\mathrm{F}$ $\left(51.63 \pm 6.41 \mathrm{~g} \mathrm{dl}^{-1}\right)$ except group $\mathrm{A}\left(53.65 \pm 0.42 \mathrm{~g} \mathrm{dl}^{-1}\right)$. But no significant $(p>0.05)$ comparative changes in $\mathrm{MCHC}$ were found in group-A, C, D, E and $\mathrm{F}$.

\subsubsection{Total leucocyte count (TLC)}

The obtained values of TLC in group A (control), B, C, D, E and F were $139.88 \pm 5.49,146.91 \pm 4.00,127.91 \pm 1.98,122.22 \pm 10.66$, $138.41 \pm 0.61$ and $140.04 \pm 2.5010^{3} \mu l^{-1}$ respectively on day 21 while on day 42 TLC value in group A (control), B, C, D, E and F were $98.27 \pm 1.37,83.97 \pm 2.36,106.59 \pm 9.94,119.46 \pm 0.80$, 
Table 3: Comparative effect of lead and different treatments on haematological profile in broiler chicken between day 21 and 42

\begin{tabular}{|c|c|c|c|c|c|c|c|}
\hline \multirow{2}{*}{$\begin{array}{l}\text { Haematological } \\
\text { parameters }\end{array}$} & \multirow[t]{2}{*}{ Day } & \multicolumn{6}{|c|}{ Various treatment groups showing mean $\pm S E$ values } \\
\hline & & A & B & C & D & $\mathrm{E}$ & $\mathrm{F}$ \\
\hline \multirow[t]{2}{*}{$\operatorname{TEC}\left(\left.10^{6} \mu\right|^{-1}\right)$} & 21 & $1.28 \pm 0.04^{\mathrm{a}}$ & $0.93 \pm 0.008^{a}$ & $1.42 \pm 0.04^{a}$ & $1.16 \pm 0.07^{\mathrm{a}}$ & $1.14 \pm 0.06^{\mathrm{a}}$ & $1.23 \pm 0.06^{\mathrm{a}}$ \\
\hline & 42 & $2.30 \pm 0.11^{b}$ & $1.82 \pm 0.11^{b}$ & $2.06 \pm 0.30^{\mathrm{a}}$ & $2.45 \pm 0.13^{b}$ & $2.44 \pm 0.26^{b}$ & $2.44 \pm 0.27^{b}$ \\
\hline \multirow[t]{2}{*}{$\mathrm{Hb}\left(\mathrm{g} \mathrm{dl}^{-1}\right)$} & 21 & $9.77 \pm 0.36^{a}$ & $7.43 \pm 0.13^{\mathrm{a}}$ & $10.44 \pm 0.23^{\mathrm{a}}$ & $11.70 \pm 1.28^{\mathrm{a}}$ & $9.60 \pm 0.09^{a}$ & $9.33 \pm 0.29^{a}$ \\
\hline & 42 & $13.30 \pm 0.64^{b}$ & $8.90 \pm 0.22^{b}$ & $12.67 \pm 1.72^{\mathrm{a}}$ & $14.66 \pm 0.90^{\mathrm{b}}$ & $15.60 \pm 2.09^{b}$ & $12.71 \pm 0.98^{\mathrm{b}}$ \\
\hline \multirow[t]{2}{*}{ PCV (\%) } & 21 & $14.47 \pm 0.41^{\mathrm{a}}$ & $9.83 \pm 0.15^{\mathrm{a}}$ & $15.43 \pm 0.29^{\mathrm{a}}$ & $13.27 \pm 0.79^{a}$ & $13.22 \pm 0.62^{\mathrm{a}}$ & $13.90 \pm 0.71^{\mathrm{a}}$ \\
\hline & 42 & $24.86 \pm 1.37^{b}$ & $16.13 \pm 0.52^{b}$ & $22.28 \pm 3.10^{\mathrm{a}}$ & $26.42 \pm 1.39^{b}$ & $26.30 \pm 2.99^{b}$ & $26.09 \pm 2.81^{\mathrm{b}}$ \\
\hline \multirow[t]{2}{*}{$\operatorname{MCV}(f l)$} & 21 & $113.09 \pm 0.61^{a}$ & $105.35 \pm 1.02^{a}$ & $109.25 \pm 2.11^{a}$ & $114.28 \pm 1.65^{\mathrm{a}}$ & $116.33 \pm 3.45^{a}$ & $113.13 \pm 1.25^{\mathrm{a}}$ \\
\hline & 42 & $107.87 \pm 1.60^{b}$ & $89.91 \pm 3.73^{b}$ & $108.87 \pm 2.30^{\mathrm{a}}$ & $107.57 \pm 0.86^{b}$ & $107.35 \pm 1.42^{\mathrm{a}}$ & $107.13 \pm 0.47^{b}$ \\
\hline \multirow[t]{2}{*}{$\mathrm{MCH}(p g)$} & 21 & $76.41 \pm 2.11^{\mathrm{a}}$ & $79.53 \pm 0.79^{a}$ & $74.91 \pm 1.80^{\mathrm{a}}$ & $101.64 \pm 11.11^{\mathrm{a}}$ & $85.26 \pm 4.44^{a}$ & $76.51 \pm 2.29^{a}$ \\
\hline & 42 & $57.83 \pm 0.61^{b}$ & $49.75 \pm 2.49^{b}$ & $62.06 \pm 2.41^{b}$ & $59.58 \pm 0.78^{b}$ & $63.03 \pm 1.79^{b}$ & $55.36 \pm 7.00^{b}$ \\
\hline \multirow[t]{2}{*}{$\operatorname{MCHC}\left(\mathrm{g} \mathrm{dl}^{-1}\right)$} & 21 & $67.54 \pm 1.60^{\mathrm{a}}$ & $75.53 \pm 1.04^{a}$ & $68.74 \pm 2.42^{\mathrm{a}}$ & $89.46 \pm 10.73^{a}$ & $73.26 \pm 3.09^{a}$ & $67.66 \pm 2.10^{\mathrm{a}}$ \\
\hline & 42 & $53.65 \pm 0.42^{b}$ & $55.29 \pm 1.21^{b}$ & $56.96 \pm 1.56^{b}$ & $55.41 \pm 0.83^{b}$ & $58.69 \pm 1.37^{b}$ & $51.63 \pm 6.41^{b}$ \\
\hline \multirow[t]{2}{*}{$\operatorname{TLC}\left(10^{3} \mathrm{dl}^{-1}\right)$} & 21 & $139.88 \pm 5.49^{a}$ & $146.91 \pm 4.00^{\mathrm{a}}$ & $127.91 \pm 1.98^{\mathrm{a}}$ & $122.22 \pm 10.66^{a}$ & $138.41 \pm 0.61^{\mathrm{a}}$ & $140.04 \pm 2.51^{a}$ \\
\hline & 42 & $98.27 \pm 1.37^{b}$ & $83.97 \pm 2.36^{b}$ & $106.59 \pm 9.94^{a}$ & $119.46 \pm 0.80^{\mathrm{a}}$ & $102.25 \pm 10.54^{b}$ & $94.56 \pm 10.70^{b}$ \\
\hline \multirow[t]{2}{*}{ Lymphocyte (\%) } & 21 & $83.10 \pm 1.84^{\mathrm{a}}$ & $52.47 \pm 1.28^{\mathrm{a}}$ & $31.27 \pm 0.17^{\mathrm{a}}$ & $41.87 \pm 3.62^{\mathrm{a}}$ & $35.77 \pm 0.80^{\mathrm{a}}$ & $35.27 \pm 2.28^{\mathrm{a}}$ \\
\hline & 42 & $22.83 \pm 1.61^{b}$ & $20.65 \pm 0.66^{b}$ & $27.63 \pm 3.58^{a}$ & $28.63 \pm 0.48^{b}$ & $23.00 \pm 2.76^{b}$ & $22.46 \pm 3.98^{\mathrm{b}}$ \\
\hline \multirow[t]{2}{*}{ Monocyte (\%) } & 21 & $6.60 \pm 0.28^{a}$ & $7.06 \pm 0.11^{\mathrm{a}}$ & $6.37 \pm 0.09^{a}$ & $7.30 \pm 0.15^{\mathrm{a}}$ & $7.00 \pm 0.09^{a}$ & $6.67 \pm 0.21^{a}$ \\
\hline & 42 & $5.13 \pm 0.32^{b}$ & $6.13 \pm 0.21^{b}$ & $5.70 \pm 0.64^{\mathrm{a}}$ & $5.16 \pm 0.31^{b}$ & $5.17 \pm 0.53^{b}$ & $4.75 \pm 0.69^{b}$ \\
\hline \multirow[t]{2}{*}{ Heterophil (\%) } & 21 & $59.16 \pm 2.24^{\mathrm{a}}$ & $54.63 \pm 0.97^{a}$ & $60.90 \pm 0.43^{\mathrm{a}}$ & $49.70 \pm 3.22^{\mathrm{a}}$ & $55.23 \pm 0.65^{\mathrm{a}}$ & $56.56 \pm 2.32^{\mathrm{a}}$ \\
\hline & 42 & $70.54 \pm 1.42^{b}$ & $71.86 \pm 1.06^{b}$ & $65.32 \pm 3.78^{\mathrm{a}}$ & $64.78 \pm 0.64^{b}$ & $70.00 \pm 2.72^{b}$ & $71.12 \pm 4.15^{b}$ \\
\hline \multirow[t]{2}{*}{ Eosinophil (\%) } & 21 & $1.00 \pm 0.37^{a}$ & $1.67 \pm 0.33^{a}$ & $1.17 \pm 0.31^{\mathrm{a}}$ & $1.00 \pm 0.52^{\mathrm{a}}$ & $1.33 \pm 0.42^{\mathrm{a}}$ & $1.17 \pm 0.31^{\mathrm{a}}$ \\
\hline & 42 & $1.00 \pm 0.37^{a}$ & $1.67 \pm 0.33^{a}$ & $0.83 \pm 0.31^{a}$ & $0.67 \pm 0.21^{a}$ & $1.17 \pm 0.31^{\mathrm{a}}$ & $1.17 \pm 0.40^{\mathrm{a}}$ \\
\hline \multirow[t]{2}{*}{ Basophil (\%) } & 21 & $0.50 \pm 0.22^{\mathrm{a}}$ & $0.67 \pm 0.21^{a}$ & $0.33 \pm 0.21^{\mathrm{a}}$ & $0.50 \pm 0.22^{\mathrm{a}}$ & $0.67 \pm 0.21^{\mathrm{a}}$ & $0.67 \pm 0.21^{\mathrm{a}}$ \\
\hline & 42 & $0.50 \pm 0.22^{\mathrm{a}}$ & $67 \pm 0.21^{\mathrm{a}}$ & $0.50 \pm 0.22^{\mathrm{a}}$ & $0.33 \pm 0.21^{a}$ & $0.67 \pm 0.33^{a}$ & $0.50 \pm 0.22^{a}$ \\
\hline
\end{tabular}

a-b Mean values bearing different superscripts within a column differ significantly $(p<0.05)$

$102.25 \pm 10.54$ and $94.56 \pm 10.7010^{3} \mu l^{-1}$ respectively. There was significant $(p<0.05)$ increased in values of TLC in lead treated group $B$ as compared to group $C, D$ and non-significant $(p>0.05)$ increase in group $A, E$ and $F$ on day 21. However comparison among treatment groups on day 21 showed significant $(p<0.05)$ decrease in the WBC count of group D from the treatment group $E$ and $F$ but group $C$ and $D$ and group $E$ and $F$ was non-significantly $(p>0.05)$ comparable to each other. Observations of day 42 revealed that TLC values were significantly $(p<0.05)$ decreased in the group A, B, E and $F$ and non-significantly $(p>0.05)$ decreased in the group $C$ and $D$ as compare to day 21 . At the end of the study lead treated group showed non-significant reduction $(p>0.05)$ from the group A (control), C, E and F but significant decrease $(p<0.05)$ from group $D$.

\subsubsection{Differential leucocyte count (DLC)}

The mean lymphocyte count was significantly $(p<0.05)$ higher in lead treated group $B(52.47 \pm 1.28 \%)$ as compared to group $A(33.10 \pm 1.84 \%), C(31.27 \pm 0.17 \%), D(41.87 \pm 3.62 \%), E$ $(35.77 \pm 0.80 \%)$ and $\mathrm{F}(35.27 \pm 02.28 \%)$ on day 21 respectively. However, on day 42 a significant $(p<0.05)$ reduction in lymphocytes number was seen in all groups except group $C$ which showed non-significant $(p>0.05)$ reduction from day 21 . At the end of experiment lead exposed (group-B) birds $(20.65 \pm 0.66 \%)$ showed non-significant $(p>0.05)$ decrease in lymphocyte count as compared to group A $(22.83 \pm 1.61 \%)$, C $(27.63 \pm 3.58 \%), D(28.63 \pm 0.48 \%), E(23.00 \pm 2.76 \%)$ and $F$ $(22.46 \pm 3.10 \%)$. Non-significant $(p>0.05)$ change was also observed in different treatment groups and was comparable to each other. Non-significant $(p>0.05)$ changes was also observed in lead treated group from the normal and other treatment groups in respect to monocytes, heterophils, eosinophils and basophils on day 21 and 42 except group $C$ which showed significant $(p<0.05)$ increased in heterophils from group $B$ and 
D while significant $(p<0.05)$ decreased monocyte from group $\mathrm{B}, \mathrm{D}$ and $\mathrm{E}$. A significant $(p<0.05)$ decrease in monocytes and heterophils count was observed in lead treated (group B) birds on day 42 as compared to its corresponding value on day 21 . However, in other groups the values remained non-significantly ( $p>0.05$ ) lower except group C.

Rahman et al. (2008) reported significant $(p<0.05)$ reduction in haemoglobin, pack cell volume, total erythrocyte count, $\mathrm{MCV}, \mathrm{MCH}, \mathrm{MCHC}$ in experimentally lead exposed broilers. These findings are in agreement with the findings of present study indicating decrease in all the haematological value in lead exposed group as there was significant $(p<0.05)$ reduction in TEC, $\mathrm{Hb}$ and PCV on day 21, and Hb, PCV and MCV on day 42 , and non significant $(p>0.05)$ decrease in $\mathrm{MCV}, \mathrm{MCH}$ and $\mathrm{MCHC}$ on day 21 and TEC, $\mathrm{MCH}$, and $\mathrm{MCHC}$ on day 42. Though at the end of the study these haematological parameters either increased significantly or non-signficantly in all the other groups except lead treated group B which showed no improvement in all the haematological values. This clearly indicated that lead had either suppressive or destructive effect on the haematopoitic activities in the body. The mechanism by which lead alters the erythrocytic parameters has been suggested by various workers. It was proposed that anaemia was the functional effect of lead poisoning due to interference with haem synthesis and decrease life span of RBCs (Waldron, 1966). Madej (1988) also suggested inhibition of haembiosynthesis, interference with porphyrine metabolism due to lead induced alterations in mitochondria and ribosome. Subsequent studies (Abou et al., 2000) in ducks also revealed marked reduction in erythrocyte count, haemoglobin $(\mathrm{Hb})$, packed cell volume (PCV\%) and mean corpuscular volume (MCV\%) which are in support of our finding. In their study Khurana et al. (1999) observed reduction in haematological parameters like total erythrocyte count, haemoglobin, packed cell volume which were decreased significantly $(p<0.05)$ in the lead exposed chicks as compared to control group chicks. Lead induced anemia was a constant finding in most of affected animals could be due to destruction of erythrocytes (due to increased fragility), depressant action on bone marrow, delaying erythrocyte maturation, and inhibition of haemsynthesis (Garg, 2000). In cases of chronic lead exposure, a microcytic, hypochromic, regenerative anemia may be present. These previous reports are in support with the present findings. Present study revealed the highest therapeutic efficacy of DL-methionine in reducing the lead induced toxic effect on TEC and Vit-E \& Se on haemoglobin followed by CQE and Vit-C. These might be due to the protective effect of antioxidants used in the present study. Earlier studies showed that Vit-E protects erythrocyte against haemolysis and it has been suggested that membrane damage resulting from the LPO is the cause of Vit-E deficiency (Chow, 1991). It appears that scavenging of free radicals by Vit-E used in the present study might have protected erythrocytes in lead exposed birds. Previous studies indicated that dietary lead increases the liver glutathione concentration (Seshadri and Khanna, 1992) and enhances the excretion of glutathione conjugates (Leeming and Donaldson, 1984). This could be correlated with low blood lead concentration, obtained in the present study, which might be due to enhanced excretion of lead in lead exposed birds, thereby protecting the erythrocytes from haemolysis. Fair et al., 2002 found that Quail (Coturnix coturnix japonica) chicks orally received either one lead shot $(0.05 \mathrm{~g})$, four lead shots $(0.2 \mathrm{~g})$, or no lead at the age of 8 days. WBC numbers increased 7 days after antigen injection on days 27 and 49 of age which is in agreement with present finding on day 21 but not with day 42 finding. In the present study the total white blood cell count was decreased non significantly $(p>0.05)$ in lead treated group B on day 42 as compared to control group. Studies by various workers have also shown leucopenia with decrease in differential leucocyte count (Avadhesh et al., 1998; Kumar et al,. 1998). On the contrary Abou et al. (2000) recorded significant $(p<0.05)$ increase in leucocyte count in ducks following lead administration for 12 weeks. The difference in the finding of present study and that of Abou et al. (2000) might be due to differences in experimental birds, dose and period of exposure. The possible mechanism by which lead induces leucopenia in birds might be due to decreased production or direct cytotoxic action of lead and increased lysis attributed to accumulation of lead (Brar et al., 1997). In the present study lymhopenia and leucopenia in lead exposed birds was observed which might be due to suppressive effect of lead on lymphoid tissues of bursal follicles. Youssef et al. (1995) have also described dose dependent lymphocytic depletion of lymphoid population in bursal follicle and spleen in lead poisoning. All the treatment groups showed least alterations in leucocytic parameters except group B indicating ameliorative potential of different therapeutic agent used in the present study.

\section{Conclusion}

It is concluded that Vit-E @ $100 \mathrm{mg} \mathrm{kg}^{-1}$ and Se @ $0.1 \mathrm{mg} \mathrm{kg}^{-1}$ having highest efficacy against lead intoxication followed by Vitamin-C @ $200 \mathrm{mg} \mathrm{kg}^{-1}$, DL-methionine @ $100 \mathrm{mg} \mathrm{kg}^{-1}$ and methanolic extract of Cissus quadrangularis (CQE) @ $400 \mathrm{mg}$ $\mathrm{kg}^{-1}$ to alleviate lead toxicosis upto the level of $200 \mathrm{mg} \mathrm{kg}^{-1}$ in broiler diet.

\section{Acknowledgement}

IB Group, Rajnandgaon, Chhattisgarh for providing free of cost VenCobb strain broiler chicks; Dean, Veterinary College Anjora, Durg (Chhattisgarh) for providing necessary facilities and Head, Department of Animal Nutrition for feed formulation.

\section{References}

Abou-Zeid, A.E., Sorour, J., Habbak, M.M., 2000. Magnitude of lead toxicity in white pekin ducklings. Egyptian Poultry Science 20(4), 789-815.

Avadhesh, K., Chauhan, R.S., Singh, N.P., Kumar, A., 1998. 
Immunopathological effect of lead on cell mediated immunity in chickens. Indian Journal of Veterinary Pathology 22(1), 22-25.

Berg, L.R., Nordstrom, J.O., Ousterhout, L.E., 1980. The preventionof chick growth depression due to dietary lead by increased dietary calcium and phosphorus levels. Poultry Science 59, 1860-1863.

Brar, R.S., Sandhu, H.S., Grewal, G.S., 1997. Biochemical alterations induced by repeated oral toxicity of lead in domestic fowl. Indian Veterinary Journal 74(5), 380-383.

Carr, A., Frei, B., 1999. Does vitamin C act as a prooxidant under physiological conditions? The Journal of the Federation of American Societies for Experimental Biology 13, 1007-1024.

Chow, C.K., 1991. Vitamin-E and oxidative stress. Free Radical Biology and Medicine 11, 215-232.

Demichele, S.J., 1984. Nutrition of lead. Comparative Biochemistry and Physiology Part A: Molecular \& Integrative Physiology 78, 401-408.

Ercal, N., Gurer-Orhan, H., Aykin-Burns, N., 2001. Toxic metals and oxidative stress. Part I: Mechanisms involved in metal-induced oxidative damage. Current Topic in Medicinal Chemistry 1(6), 529-539.

Fair, J.M., Ricklefs, R.E., 2002. Physiological, growth, and immune responses of japanese quail chicks to the multiple stressors of immunological challenge and lead shot. Archive of Environmental Contamination and Toxicology 42(1), 77-87.

Garg, S.K., 2000. Toxicity of Metals ( $1^{\text {st }}$ Edn.). \CBS Publishers and Distributors, New Delhi, 42-47.

Heaney, R.P., 2000. Lead in calcium supplements. Journal of the American Medical Association 284, 1432-1433.

Khan, M.S.H., Mostofa, M., Jahan, M.S., Sayed, M.A., Hossain, M.A., 2008. Effect of garlic and vitamin-B complex in lead acetate induced toxicities in mice. Bangladesh Journal of Veterinary Medicine 6(2), 203-210.

Khurana, S.K., Chauhan, R.S., Mahipal, S.K., 1999. Haematological changes in chronic lead intoxication in chickens. Indian Journal of Veterinary Pathology
23-93.

Kucuk, O., Sahin, N., Sahin, K., Gursu, M.F., Gulcu, F., Ozcelik, M., Issi, M., 2003. Egg production, egg quality and lipid peroxidation status in laying hens maintained at a low ambient temperature and fed a vitamin C and vitamin $\mathrm{E}$ supplemented diet. Veterinary Medicine Czech 2, 33-40.

Kumar, A., Chauhan, R.S., Singh, N.P., 1998. Immunopathological effect of lead on cell mediated immunity in chickens. Indian Journal of Veterinary Pathology 22, 22-25.

Lee, W.R., 1981. What happens in lead poisoning? Journal of the Royal College of Physicians of London, 15, 48-54.

Leeming, T.K., Donaldson, W.E., 1984. The effect of dietary methionine and lysine on the toxicity of ingested lead acetate in the chick. Journal of Nutrition 114(11), 2155-2159.

Madej, J.A., Radzanowska, G., Klimentowski, S., Zarka, M., 1988. The action of some haematological and biochemical indices and the histopathological changes in the organ of hen given lead, selenium or lead plus selenium by mouth. Weterynaria 44 (172), 113-127.

Osweiler, G.D., 1996. Toxicology. Wilians and Wilkins, Philadelphia, 191-197.

Rahman, S., Bhojane, N.M., Pathak, V.P., Ranvir, G.D., Joshi, M.V., 2008. Haematobiochemical observations in experimental lead toxicity in broilers. The Indian Veterinary Journal 85, 930-933.

Seshadri, S., Khanna, A., 1992. Changes in the tissue concentration of the glutathione and protein in lead toxicity. Current Science 51, 510-513.

Snedecor, G.W., Cochran, W.G., 1994. Statistical methods ( $8^{\text {th }}$ Edn.). lowa State Univ Press, Ames, IOWA2.

Waldron, H.A., 1966. The anaemia of lead poisoning: a review. British Journal of Industrial Medicine 23, 83-100.

Youssef, S.A.H., Sanousi, A.A., Afifi, N.A., Brawy, A.L., 1996. Effect of subclinical toxicity on the immune response of chicken to new castle disease virus vaccine. Research in Veterinary Science 60, 13-16. 